HUTP-99/A020

NUB 3197

EFI-99-12

June 30, 1999

\title{
Higgs as a Slepton
}

\author{
Aaron K. Grant ${ }^{1}$ and Zurab Kakushadze ${ }^{1,2,3}$ \\ ${ }^{1}$ Jefferson Laboratory of Physics, Harvard University, Cambridge, MA 02138 \\ ${ }^{2}$ Department of Physics, Northeastern University, Boston, MA 02115 \\ ${ }^{3}$ Enrico Fermi Institute, University of Chicago, Chicago, IL 60637 \\ grant@gauss.harvard.edu,zurab@string.harvard.edu
}

\begin{abstract}
In theories with $\mathrm{TeV}$ scale quantum gravity there is a logical possibility where the electroweak Higgs can be a fourth generation slepton. Despite maximal supersymmetry breaking such a scenario turns out to be tightly constrained, and might be testable already at present collider experiments. Also, a light Higgs is no longer a prediction of supersymmetry.
\end{abstract}


Recently it was suggested that the fundamental scale of quantum gravity $M_{P f}$ may be as low as a few $\mathrm{TeV}$, which would provide an alternative way of understanding the hierarchy problem [1]. In this proposal the observed weakness of gravity at large distances is due to the presence of $n$ large new dimensions (of size $\sim R>>M_{P f}^{-1}$ ) in which gravity can propagate. The relation between the observed Planck scale $M_{P}$ and the fundamental Planck scale $M_{P f}$ is given by

$$
M_{P}^{2} \sim M_{P f}^{n+2} V_{n}
$$

where $V_{n} \sim R^{n}$ ( $n$ must be 2 or larger) is the volume of the $n$ extra spatial dimensions (i.e., of the bulk). In this picture all the Standard Model fields must reside inside a brane (or a set of branes) with 3 extended spatial dimensions f A general discussion of possible embeddings of such a scenario within the string theory context was given in [4, 5, 6].

In this framework the role of supersymmetry might be somewhat modified. $A$ priori it is no longer needed as a solution to the hierarchy problem. However, it might still play an important role in, say, formulating the fundamental theory that incorporates quantum gravity above a $\mathrm{TeV}$. At present the only known candidate theory for such a unified picture is superstring theory. Thus, we may expect string theory to take over at energies above a $\mathrm{TeV}$ with supersymmetry broken not much below the fundamental string scale $M_{s}$. If so, what is the content of the supersymmetric theory just below $M_{s}$ where stringy modes can be integrated out?

The purpose of this note is to point out that in theories with quantum gravity (or strings) at the TeV scale, there is a logical possibility to identify the electroweak Higgs with the scalar superpartner of one of the lepton doublets (or a linear combination thereof). However, we cannot identify the $S U(2)$-breaking slepton with the superpartner of a known lepton. Rather, we propose the existence of a fourth generation, whose slepton plays the role of the Higgs boson. In this way, the Higgs and flavor structures of the theory are more closely related than in the usual supersymmetric standard model. Other proposals have been made wherein a sneutrino belonging to one of the first three generations has a small $S U(2)$-breaking vacuum expectation value [0]. Our proposal differs in that the fourth generation sneutrino is the sole source of $S U(2)$ breaking.

Already in the early days of supersymmetric model building, the intriguing similarity of the quantum numbers of the weak lepton doublets $\left(L^{a}\right)$ and the electroweak Higgs $H$ made it tempting to speculate that the Higgs might actually be the superpartner of a lepton (that is, a slepton). Unfortunately, within the usual paradigm with the fundamental Planck scale $\sim 10^{19} \mathrm{GeV}$ this is impossible because of the well known rules for writing supersymmetric Lagrangians. Thus, the couplings

$$
L^{*} Q U_{c}
$$

\footnotetext{
${ }^{1}$ In a somewhat different context an attempt to lower the string scale down to a $\mathrm{TeV}$ (without lowering the fundamental Planck scale) was considered in [2] based on an earlier observation in [3].
} 
which in these scenarios are required to give masses to the up-type quarks, would be suppressed by the factor $M_{\text {susy }}^{2} / M_{P}^{2}$, which can at best be $\sim 10^{-32}$ or so.

However, the situation is dramatically different if the fundamental Planck scale is around a TeV. Now the desired couplings of the slepton Higgs to the fermions can be generated through the couplings in the Kähler potential

$$
\int d^{4} \theta g_{a b}^{u} \frac{X^{*} L^{*}}{M_{P f}^{2}} Q^{a} U_{c}^{b},
$$

where $X$ is the "spurion" superfield which breaks supersymmetry through the Fterm $\left\langle F_{X}\right\rangle \sim \theta^{2} M_{P f}^{2}$. Then the above terms generate effective Yukawa couplings of the form $g_{a b}^{u} L_{\text {scalar }}^{*} Q_{\text {fermion }}^{a} U_{\text {c,fermion, }}^{b}$, where $L$ is the lepton superfield whose scalar component is identified with the Higgs, and $U_{c}$ is the charge-conjugated up-type quark. Note that in this context no additional suppression appears. The couplings that generate masses of the down quarks and charged leptons can come (depending on the details of a given model) from the superpotential (as usual)

$$
\Delta W=g_{a b}^{d} L Q^{a} D^{b}+g_{a b}^{e} L L^{a} e_{c}^{b},
$$

and/or from the Kähler potential

$$
\Delta K=\frac{X^{*}}{M_{P f}^{2}}\left(g_{a b}^{d} L Q^{a} D^{b}+g_{a b}^{e} L L^{a} e_{c}^{b}\right) .
$$

Small neutrino masses (if they are non-zero) are more subtle and may involve intrinsically higher dimensional mechanisms [8] which will not be discussed here. Here we should point out that the mass of the fermionic component of $L$ (that is, of the lepton superfield whose scalar component is identified with the Higgs) cannot be generated from the above couplings. Indeed, couplings $L L e_{c}^{a}$ and $X^{*} L L e_{c}^{a}$ vanish due to the antisymmetry of the weak $S U(2)$ contractions. However, there are other terms in the Kähler potential that give rise to the desired Yukawa couplings $L_{\text {scalar }} L_{\text {fermion }} e_{\text {c,fermion. }}^{a}$ For instance, consider the term

$$
\frac{(\mathcal{D} X) X^{*}}{M_{P f}^{4}} L(\mathcal{D} L) e_{c}^{a},
$$

where $\mathcal{D}$ denotes the covariant superderivative. In fact, if there is some hierarchy between the supersymmetry breaking scale and $M_{P f}$, then this might be (partially) responsible for the fermion mass hierarchy between the quark and lepton sectors.

A priori within this framework there might arise problems associated with lepton flavor violation. However, this problem might be solved by introducing appropriate flavor symmetries. We specialize to the case where the electroweak Higgs is identified with a fourth generation slepton $L^{4}$. (It will become clear momentarily why we cannot identify the Higgs boson with the superpartner of a known neutrino.) Then 
the couplings (田) that generate charged lepton masses will also generate the couplings of the form

$$
g_{a b}^{e} L_{\text {fermion }}^{4} L_{\text {scalar }}^{a} e_{\text {c,fermion }}^{b},
$$

which will generically induce various flavor violating transitions. The same will be true if masses are generated from (5). Thus, we have to impose flavor symmetries. For instance, we can attempt to impose individual $e_{-}, \mu$ - and $\tau$-lepton number symmetries. This would guarantee that the individual lepton numbers are conserved. Alternatively, we may attempt to solve the flavor violation problem along the lines of [9, 10] $]^{2}$. Here we should point out that the baryon number violating terms must be adequately suppressed to avoid too rapid proton decay. Generically this requires imposing additional symmetries (see, e.g., [12]). However, it appears that this can be done consistently with generating acceptable fermion masses in the above framework 13.

The spectrum of new particles in this class of models differs from that found in the minimal supersymmetric standard model. In particular, the model has only a single Higgs boson and only three neutralinos. The charginos of the model are a linear combination of the charged gauginos and the fourth generation lepton. Their mass matrix has the form

$$
\left(\begin{array}{cc}
M_{2} & 0 \\
\sqrt{2} m_{W} & m_{4}
\end{array}\right)
$$

in the $\left(\widetilde{W}, L_{4}\right)$ basis, where $M_{2}$ is the $S U(2)$ gaugino mass, and $m_{4}$ is the fourth generation lepton mass. The neutralinos are mixtures of the neutral gauginos and the fourth generation neutrino. In the $\left(\widetilde{B}, \widetilde{W}^{3}, \nu_{4}\right)$ basis, their mass matrix has the form

$$
\left(\begin{array}{ccc}
M_{1} & 0 & -m_{Z} \sin \theta_{W} \\
0 & M_{2} & m_{Z} \cos \theta_{W} \\
-m_{Z} \sin \theta_{W} & m_{Z} \cos \theta_{W} & 0
\end{array}\right) .
$$

We can now explain why it is impossible to identify the Higgs boson with the superpartner of a known neutrino. Indeed, had we identified the Higgs doublet with, say, the tau lepton doublet, then we would find that tau neutrino typically acquires a see-saw type mass of order $m_{Z}^{2} / M_{1,2}$. This mass, of order $10 \mathrm{GeV}$, is far too large. Hence we choose the alternate route of introducing a fourth generation, and identifying its sneutrino with the Higgs boson.

Limits from collider searches for new particles constrain the parameter space of this class of models. The charginos have masses that can exceed LEP-II collider limits [14, provided that $M_{2}$ and $m_{4}$ are sufficiently large. However, the neutralinos cannot be made arbitrarily heavy, and indeed this class of models always has at least one relatively light neutralino. As an example, take the values

$$
M_{1}=45 \mathrm{GeV}, \quad M_{2}=110 \mathrm{GeV}, \quad m_{4}=185 \mathrm{GeV} .
$$

\footnotetext{
${ }^{2}$ Fermion Yukawa hierarchy from flavor symmetry breaking on distant branes [1] can also be combined with this scenario.
} 
We then have $m_{\chi_{1,2}^{+}}=90,226 \mathrm{GeV}$, while the neutralino masses are

$$
m_{\chi_{1}^{0}}=56 \mathrm{GeV}, \quad m_{\chi_{2}^{0}}=57 \mathrm{GeV}, \quad m_{\chi_{3}^{0}}=156 \mathrm{GeV} .
$$

The lightest neutralino is predominantly $U(1)$ gaugino, the next to lightest is mostly neutrino, and the heaviest is mostly $S U(2)$ gaugino. In $e^{+} e^{-}$collisions at $\sqrt{s}=183$ $\mathrm{GeV}$, the production cross sections for the lightest two neutralinos are $0.002 \mathrm{pb}$ and $0.31 \mathrm{pb}$ respectively, provided that the sfermions are heavy so that $Z$ exchange is the dominant production mechanism. The relative size of the cross sections is easy to understand, since the lightest neutralino is mostly bino and therefore couples very weakly to the $Z$. In this particular case, neutralino production will be dominated by $\chi_{2}^{0}$ pairs. The $\chi_{2}^{0}$ may either decay promptly to three-body final states, or may decay first to $\chi_{1}^{0} Z^{*}$ or $\chi_{1}^{0} \tilde{\nu}_{4}^{*}$, leading to five (or more)-body final states. The decays of the $\chi_{1,2}^{0}$ are highly model dependent, but we can delineate the main possibilities.

1. R-parity is strictly preserved for the first three generations, and broken only by the Yukawa couplings of the fourth generation lepton. Here we have two possibilities, depending on the mass of the gravitino $\tilde{G}$. If decays to the gravitino are allowed, we expect decays like $\chi_{1,2}^{0} \rightarrow \bar{f} f \tilde{G}$, where $f$ can be a lepton or quark. If decays to the gravitino are kinematically forbidden, the lightest neutralino is absolutely stable. In this case, $\chi_{2}^{0}$ pair production will result in events with rather soft jets and leptons coming from the $\chi_{2}^{0} \rightarrow \chi_{1}^{0} Z^{*}, \chi_{1}^{0} \tilde{\nu}_{4}^{*}$ decay. Also note that the lightest neutralino in this case is the SUSY dark matter candidate.

2. If R-parity is broken for all four generations, then $\chi_{1,2}^{0}$ will decay to the standard model particles. The decays are mediated by couplings of the form $L L e_{c}$ or $L Q D$, where the superfields can belong to any of the four generations. The cross section for $\chi_{2}^{0}$ pair production for the sample point given above is 0.31 $\mathrm{pb}$. This exceeds the experimental limit of roughly $0.1 \mathrm{pb}$ [14] if the neutralino decays primarily to leptonic final states via $L L e_{c}$ couplings. Hence the neutralinos must decay primarily to hadronic final states via couplings like $L Q D$. In this case, the cross section limits are about $0.6 \mathrm{pb}$ [14], and the sample point is not excluded. Hence we expect decays like $\chi^{0} \rightarrow q \tilde{q}^{*} \rightarrow q q \ell$, so that the final states for $\chi_{2}^{0}$ pair production will contain 4 jets together with either charged leptons or missing energy if the $\chi_{2}^{0}$ decays promptly. If it decays first to $\chi_{1}^{0} Z^{*}$ or $\chi_{1}^{0} \tilde{\nu}_{4}^{*}$ there will be additional particles in the final state from $Z, \tilde{\nu} \rightarrow f \bar{f}$. Conservative limits on the dimensionless coefficients of $L Q D$ couplings among the first three generations are at the level of 0.4-0.001 [15]. Under less conservative assumptions, tighter constraints can be derived [16]. For instance, bounds from neutrinoless double beta decay [17] imply $\lambda_{113}^{\prime} \lambda_{131}^{\prime}<8 \times 10^{-8}$. Hence it seems not unlikely that the $b$-quark Yukawa coupling $L_{4} Q_{3} D_{3}$ is among the largest of the $\mathrm{R}$-parity violating terms that can contribute to neutralino decay. If this is the case, the final states will frequently include $b$-flavored hadrons. 
Although we have illustrated the qualitative features of this scenario for a particular point in parameter space, certain predictions are fairly model independent. In particular, we expect that models of this type will always possess at least one neutralino with mass below $65 \mathrm{GeV}$. Indeed, varying $M_{1}$ and $M_{2}$ between 0 and 1 $\mathrm{TeV}$, we find that the neutralino mass matrix always has at least one eigenvalue below $65 \mathrm{GeV}$. Also, we note that the neutralino production cross section is not likely to be too small. The cross section from $Z$ exchange, summed over neutralino species, is larger than $0.23 \mathrm{pb}$ for all points in parameter space where $M_{1,2} \leq 1 \mathrm{TeV}$, where charginos cannot be pair produced at LEP-II, and where $Z$ decays to pairs of neutralinos are kinematically forbidden. The cross section could be smaller if there are large cancellations between $Z$ exchange and $t$-channel sfermion exchange. If we permit lighter charginos, then of course we expect still more light particles and a richer phenomenology. We have emphasized the case of heavy charginos and lighter neutralinos since constraints on R-parity violating charginos are quite stringent [14].

We would like to end this note by summarizing some of the model independent predictions of the above scenario.

1. R-parity is necessarily broken by the Yukawa couplings. As a result, the production and decay of superpartners is modified compared to what one finds in R-parity conserving models. We have outlined the most novel aspects of this scenario above in connection with neutralino pair production. Apart from this, one expects the usual signatures of R-parity violating supersymmetry, such as the absence of missing energy in SUSY particle decays.

2. A light Higgs is no longer a robust prediction of supersymmetry. This is much in the spirit of 18 where it was pointed out that $M_{P f}$ suppressed operators can ameliorate an upper bound on the Higgs mass in the non-supersymmetric context. In our case the Higgs potential can come from the Kähler potential

$$
\frac{X^{*} X}{M_{P f}^{2}}\left(L L^{*}+\frac{a}{M_{P f}^{2}}\left(L L^{*}\right)^{2}+\ldots\right),
$$

which can in principle tolerate any mass the Higgs could have in the context of the non-supersymmetric Standard Model.

3. Although we no longer necessarily expect a light Higgs, we do in general expect one or more light neutralinos, with mass less than $65 \mathrm{GeV}$. If the charginos are too heavy to be pair produced at LEP-II, then at least one of the neutralinos is likely to have a production cross section of $0.25 \mathrm{pb}$ or larger, which is not too far from present experimental limits.

Finally, let us point out that the entire scheme discussed in this note is possible because supersymmetry in the context of $\mathrm{TeV}$ scale gravity is broken softly but (almost) completely. Thus, for instance, the Yukawa couplings not allowed by supersymmetry are generated via higher dimensional operators in the Kähler potential 
once supersymmetry is broken. On the other hand, we have shown that the underlying supersymmetry leads to non-trivial predictions in this scenario, such as the presence of light neutralinos. It is conceivable that this scenario can be confirmed or excluded by LEP-II or the Tevatron.

We would like to thank Gia Dvali for collaboration at initial stages of this work and valuable discussions. We would also like to thank Howard Georgi, Henry Tye, and Cumrun Vafa for useful discussions. The work of A.K.G. was supported by NSF grant PHY-9802709. The work of Z.K. was supported in part by NSF grant PHY-96-02074, and the DOE 1994 OJI award. This work was completed during Z.K.'s visit at the Enrico Fermi Institute. Z.K. would also like to thank Albert and Ribena $\mathrm{Yu}$ for financial support.

\section{References}

[1] N. Arkani-Hamed, S. Dimopoulos and G. Dvali, Phys. Lett. B429 (1998) 263; Phys. Rev. D59 (1999) 086004.

[2] J. Lykken, Phys. Rev. D54 (1996) 3693.

[3] E. Witten, Nucl. Phys. B471 (1996) 135.

[4] I. Antoniadis, N. Arkani-Hamed, S. Dimopoulos and G. Dvali, Phys. Lett. B436 (1998) 257.

[5] G. Shiu and S.-H.H. Tye, Phys. Rev. D58 (1998) 106007.

[6] Z. Kakushadze and S.-H.H. Tye, Nucl. Phys. B548 (1999) 180.

[7] C. Liu and H.S. Song, "MeV tau neutrino in gauge mediated supersymmetry breaking model," Nucl. Phys. B545, 183 (1999) hep-ph/9811203, and earlier references therein.

[8] N. Arkani-Hamed, S. Dimopoulos, G. Dvali and J. March-Russell, talk presented by S. Dimopoulos at SUSY98, hep-ph/9811448;

K.R. Dienes, E. Dudas and T. Gherghetta, hep-ph/9811428;

G. Dvali and A.Yu. Smirnov, hep-ph/9904211.

[9] Z. Berezhiani and G. Dvali, Phys. Lett. B450 (1999) 24.

[10] Z. Kakushadze, Nucl. Phys. B551 (1999) 549.

[11] N. Arkani-Hamed and S. Dimopoulos, hep-ph/9811353.

[12] Z. Kakushadze, Nucl. Phys. B548 (1999) 205; hep-th/9812163.

[13] Work in progress. 
[14] ALEPH Collaboration, R. Barate et al., hep-ex/9811033;

ALEPH Collaboration, R. Barate et al., hep-ex/9712013;

L3 Collaboration, M. Acciarri et al., preprint CERN-EP/99-023;

OPAL Collaboration, G. Abbiendi et al., hep-ex/9901037.

[15] H. Dreiner, in "Perspectives on Supersymmetry", ed. G.L. Kane (World Scientific, Singapore, 1998), hep-ph/9707435.

[16] See, e.g.,

R. Barbier et al., hep-ph/9810232, and earlier references therein.

[17] K.S. Babu and R.N. Mohapatra, Phys. Rev. Lett. 75 (1995) 2276;

M. Hirsch and H.V. Kladpor-Kleingrothaus, Nucl. Phys. Proc. Suppl. 62B (1998) 224.

[18] L. Hall and C. Kolda, hep-ph/9904236. 